\title{
The trainees' pain with laparoscopic surgery: what do trainees really know about theatre set-up and how this impacts their health
}

\author{
Declan Quinn • James Moohan
}

Received: 31 May 2014 / Accepted: 24 November 2014 / Published online: 4 December 2014

(C) Springer-Verlag Berlin Heidelberg 2014

\begin{abstract}
Although it is clear that laparoscopic surgery is beneficial to the patient, such surgery brings with it unique challenges and possible injury to the surgeon. Firstly, we sought to investigate the prevalence of musculoskeletal distress experienced by trainees. Secondly, we sought to ascertain if the trainees had received appropriate instruction to optimise their operative environment during laparoscopic surgery. An anonymised questionnaire survey was distributed to all 89 trainees in obstetrics and gynaecology within Northern Ireland. Forty-four ( $83 \%)$ trainees reported to having received formal instruction in theatre layout and operating body position. However, only $8(15 \%)$ were aware of the ideal operating surface height, and $6(11 \%)$ knew the ideal monitor position, while $11(20 \%)$ and $7(13 \%)$ knew the correct angles for grasping and suturing tissue, respectively. Eightyfive percent of trainees suffered some form of musculoskeletal distress with back, shoulder and neck pain the most common areas affected. Eyestrain was reported by $1 / 3$ of trainees. Although no trainees required sick leave, one in three required regular analgesia, physiotherapy or alternative therapies. It is clear that current training has not addressed operating ergonomics sufficiently, and this is having a significant impact on trainees' health.
\end{abstract}

Keywords Laparoscopy · Ergonomics · Gynaecology · Training

D. Quinn $(\bowtie)$

ST6 Antrim Area Hospital, 4 Bush Road, Antrim, NI, UK

e-mail: quinndeclan@hotmail.com

J. Moohan

Altnagelvin Area Hospital, Londonderry, UK

e-mail: James.moohan@westerntrust.hscni.net

\section{Background}

Among surgeons, musculoskeletal symptoms have been reported to increase in prevalence with increasing age [1]. In addition, Szeko et al., (2009) noted that the likelihood of musculoskeletal pain was closely related to the number of years worked in laparoscopic surgery [2]. In contrast, Sari et al. (2010) found that musculoskeletal symptoms were more prevalent in surgeons with less experience [3], while Soueid et al. (2010) found that such symptoms were often experienced at an early age [4]. Park et al. (2010) however found no relationship between musculoskeletal symptoms and age [5].

The elongated duration of the laparoscopic surgical procedure compared with the equivalent open procedure also appears to play a role in the prevalence of musculoskeletal distress among surgeons [6]

To date, little or no attention has been paid to the impact that the increasing proportion of surgery performed laparoscopically has had on the incidence of musculoskeletal distress among trainees.

The objectives of this study were to examine the prevalence of eyestrain and musculoskeletal distress among trainee gynaecologists and to ascertain whether instruction in theatre and surgical ergonomics had any impact on this.

\section{Method}

A survey in the form of a questionnaire was distributed to trainees in obstetrics and gynaecology of the Northern Ireland Deanery attending their monthly CME meetings between 1 September 2012 and 31 December 2012. A list of the email addresses of each trainee was obtained from the Northern Ireland Medical and Dental Training Agency, to enable a copy of the questionnaire survey to be sent electronically to any trainee who had either failed to complete a questionnaire at the 
Table 1 Group demographics

\begin{tabular}{llll}
\hline & Male & Female & $\begin{array}{l}\text { Between-group } \\
\text { statistics }\end{array}$ \\
\hline Age & $33(27-55)$ & $29(25-38)$ & $<0.05$ \\
Height $(\mathrm{cm})$ & $178.3(167-188)$ & $164.5(153-180)$ & $<0.01$ \\
Weight $(\mathrm{kg})$ & $86.3(68-110)$ & $61.4(47-49)$ & $<0.01$ \\
Glove size & $7.5(5.5-8.5)$ & $6(5.5-7.0)$ & $<0.01$ \\
Trainee ST level & $3.0(1-7)$ & $3.0(1-7)$ & $\mathrm{ns}$ \\
Years in specialty & $5.6(1-31)$ & $3.9(1-10)$ & $\mathrm{ns}$ \\
\hline
\end{tabular}

Data are presented as group mean values (range)

meeting or who had been unable to attend. Each questionnaire was accompanied by a letter explaining the aims of the study and included a consent form.

\section{Survey design}

The survey examined information in three categories:

1. Basic demographics of each trainee

2. The degree of instruction and knowledge on the ergonomics of gynaecological endoscopic surgery

3. The prevalence/frequency ${ }^{1}$ and sites of possible musculoskeletal distress suffered and the requirement for treatment of such injury

\section{Data analysis}

The data obtained from the questionnaire were analysed using SPSS version 20. Basic demographics were assessed using descriptive statistics with male and female groups being compared using the independent sample $t$ test. The chi-square test for independence was employed to compare the prevalence of musculoskeletal distress and eyestrain between the two sexes with the Yates correction for continuity being used to compensate for any overestimation in the chi value that results from a $2 \times 2$ table. Collinearity diagnostics were employed to exclude the possibility of high correlation between different variables before binary logistic regression was employed to determine whether different independent variables were able to predict the prevalence of musculoskeletal distress or eyestrain.

\footnotetext{
${ }^{1}$ The frequency of musculoskeletal distress was rated on a Likert scale (always, frequently, occasionally, rarely and never).
}

\section{Findings}

Fifty-three out of 89 trainees completed the questionnaire to give a response rate of $60 \%$. Nineteen of the trainees were male $(36 \%)$ with the remainder $(64 \%)$ being female, a ratio in keeping with the local medical school graduate output.

The median age for the group as a whole was 30 years (range 25-55) with males being somewhat older (median age 30 versus 29 years). As expected, male trainees were taller, heavier and had a larger hand size than their female counterparts (Table 1). In addition, although male and female trainees were at the same level of specialist training, male trainees had on average spent almost 2 years longer within the specialty (Table 1).

The majority of trainees experienced some degree of musculoskeletal distress with $1 / 3$ suffering eyestrain (Table 2).

Although male trainees suffered a lower prevalence of both injuries when compared with females, a chi-square test for independence indicated no significant association between gender and injury (Yates correction for continuity $=0.256$, $p=0.613$ and $0.332, p=0.564$ for musculoskeletal distress and eyestrain, respectively). All levels of trainees were found to suffer symptoms. Surprisingly, although one might have anticipated that senior trainees would perform a greater amount of laparoscopic surgery as well as more complex procedures than their junior colleagues and thus be more likely to experience injury, a chi-square test for independence revealed that they were no more likely to suffer symptoms (Pearson chi-square $=4.575, p=0.599$, $\mathrm{phi}=0.249$ ).

Twenty-two trainees admitted to having received some form of instruction in the ergonomics of laparoscopic surgery with more than half of male trainees (11 of 19, $58 \%$ ) receiving instruction compared to only 1 in 3 female trainees (11 of 34, $32 \%$ ), but again, this difference was not significant (Yates

Table 2 Prevalence of pain and eyestrain between groups

\begin{tabular}{llll}
\hline & Male & Female & Total \\
\hline Pain during laparoscopy & $15(78.9 \%)$ & $30(88.2 \%)$ & $45(84.9 \%)$ \\
Eyestrain & $5(26.3 \%)$ & $13(38.2 \%)$ & $18(34 \%)$ \\
\hline
\end{tabular}


Table 3 Ergonomic instruction and prevalence of pain

\begin{tabular}{llll}
\hline & Ergonomic instruction & Musculoskeletal pain & Eyestrain \\
\hline \multirow{2}{*}{ Male } & Yes & 9 & 2 \\
& No & 5 & 3 \\
\multirow{2}{*}{ Female } & Yes & 9 & 4 \\
& No & 21 & 9 \\
\hline
\end{tabular}

correction for continuity $=2.308, p=0.129, \mathrm{phi}=0.249$ ). The source of instruction on ergonomics was most often a course $(11,50 \%)$, a senior colleague $(3,13.6 \%)$ or both $(7,31.8 \%)$.

Instruction on ergonomics however did not result in a significant reduction in the likelihood of musculoskeletal distress or eyestrain (Yates correction for continuity $=0.019, p=0.889$ and $0.327, p=0.567$ for musculoskeletal pain and eyestrain, respectively, for the whole group of trainees). Indeed, 9 of 11 male trainees and 9 of 11 females who had received some degree of instruction still experienced musculoskeletal pain (Table 3).

Eighteen (33\%) trainees reported suffering eyestrain, with male trainees ( 5 of 19, $26 \%$ ) again being affected less often than their female counterparts ( 13 of $34,38 \%$ ). Instruction on ergonomics does appear to reduce the incidence of eyestrain but not significantly, with only $27.3 \%$ of trainees who had received instruction being affected compared to $38.7 \%$ of those who had not. Among male trainees, the impact of instruction appears even greater with only 2 of $11(18 \%)$ suffering eyestrain compared to 3 of $8(37.5 \%)$ who did not receive such teaching. Four of 11 (36\%) female trainees suffered eyestrain despite having received instruction similar to the proportion of trainees who had received no instruction (9 of 23, $39 \%$ ). A chi-square test for independence did not reveal any significant difference between the sexes (Yates correction for continuity $=0, p=0.72$ and $=0.055, p=0.815$ for male and female, respectively). A larger study would hopefully confirm or refute these findings.

Pain occurred most commonly in the neck (42\%), back (72\%), shoulder (43\%) or leg (37\%), occurring less frequently in the elbow, wrist, thumb, fingers and foot (Table 4).
Table 5 Prevalence of pain and direction of neck turning between the groups

\begin{tabular}{llllll}
\hline & Neutral & Left & Right & Both & All directions \\
\hline Male & 1 & 1 & 3 & 1 & 0 \\
Female & 5 & 2 & 4 & 4 & 1 \\
Total & 6 & 3 & 7 & 5 & 1 \\
\hline
\end{tabular}

For each site of pain, there was no significant difference in prevalence between male and female trainees (Table 4).

Neck pain occurred most commonly on turning to the right ( 7 of $22,31 \%$ ) followed by being in the neutral position ( 6 of $22,27 \%$ ) with pain being reported as occurring frequently in $1 / 3$ trainees and occasionally in $2 / 3$ trainees, male and female alike. (Table 5)

With regard to back pain, this was just as likely to occur in the region of the lower as upper back, with female trainees being more likely to suffer pain in both areas when compared to their male counterparts (Table 6) although a chi-square test for independence did not detect any significant difference between the sexes (Pearson chi-square $=2.724, p=0.256$ ).

Male trainees suffered back pain frequently in three cases $(21.4 \%)$ compared to female trainees who experienced the pain frequently in eight cases $(33.3 \%)$, but again, this did not reach significance.

Shoulder pain occurred slightly more often on the right side ( 9 of $23,39.1 \%$ ) with both shoulders being affected in more than 8 trainees $(34.7 \%)$. The dominance of rightsided injury was only apparent among female trainees $(7 / 16,43.8 \%)$ with male counterparts actually experiencing pain more commonly in the left shoulder. However, this difference did not reach statistical significance (Yates correction for continuity $=0.186, p=0.667$ ). Pain was reported as occurring frequently in 5 trainees $(21.7 \%)$ and occasionally in the remainder with female trainees being twice ( 25 versus $14.2 \%$ ) as likely to be affected frequently as males, but again, this did not reach significance (Pearson chi-square $=1.483, p=0.476$ ).
Table 4 Site of pain between the groups and significance

\begin{tabular}{lrrlrrr}
\hline Site of pain & Male & Female & $\begin{array}{l}\text { Continuity } \\
\text { correction }\end{array}$ & $p$ & Phi & $\begin{array}{l}\text { Approximate } \\
\text { significance }\end{array}$ \\
\hline Neck & 6 & 16 & 0.650 & 0.420 & 0.151 & 0.279 \\
Back & 14 & 24 & 0.000 & 1.000 & -0.033 & 0.810 \\
Shoulder & 7 & 16 & 0.186 & 0.667 & 0.099 & 0.472 \\
Elbow & 3 & 5 & 0.000 & 1.000 & -0.015 & 0.916 \\
Wrist & 2 & 9 & 1.039 & 0.308 & 0.189 & 0.170 \\
Thumb & 5 & 7 & 0.018 & 0.892 & -0.066 & 0.433 \\
Finger & 5 & 6 & 0.155 & 0.461 & -0.103 & 0.456 \\
Leg & 7 & 13 & 0.000 & 1.000 & 0.014 & 0.920 \\
Foot & 5 & 6 & 0.155 & 0.461 & -0.103 & 0.456 \\
\hline
\end{tabular}


Table 6 Site of back pain between the groups

Leg pain affected male and female trainees in similar numbers ( 36.8 and $38.2 \%$, respectively) and usually affected both legs $(13 / 20)$. Furthermore, leg pain was usually only experienced occasionally $(15 / 20,75 \%)$ rather than frequently $(3 / 20,9 \%)$.

Two trainees, both female, had required sick leave as a result of the pain experienced, while one trainee, also female, had sought medical attention, but again, the difference between sexes was not significant (Yates correction for continuity $=0.106, p=0.744$ for sick leave and Yates correction for continuity $=0.000, p=1.000$ for medical attention). Seventeen trainees had however resorted to some form of treatment for their symptoms (Table 7).

Surprisingly, trainees who had received instruction in ergonomics were no more likely to answer correctly those questions (Table 8) pertaining to optimal ergonomics for gynaecological laparoscopy, such as the ideal height of the operating surface (Yates correction for continuity $=3.191, p=$ 0.784 ), ideal height of the centre of the monitor (Yates correction for continuity $=7.393, p=0.117$ ), ideal instrument angle for dissection/grasping (Yates correction for continuity $=5.671, p=0.340$ ) or suturing (Yates correction for continuity $=6.892, p=0.199$ ), than those trainees who had not received instruction (Table 8). Again, there was no significant difference between male (Pearson chi-square $=2.943$, $5.589,8.573$ and $4.369 ; p=0.709,0.232,0.073$ and 0.358 for operating height, monitor position, ideal dissection/ grasping and ideal suturing angle, respectively) and female trainees (Pearson chi-square $=6.497,1.526,2.807$ and 4.369; $p=0.370,0.676,0.730$ and 0.629 for operating height, monitor position, ideal dissection/grasping and ideal suturing angle, respectively).

After collinearity diagnostics had been performed to exclude high correlation between different variables within the model, direct logistic regression was performed to assess the

Table 7 Interventions required between the groups

\begin{tabular}{lllc}
\hline & Male & Female & Total \\
\hline Analgesia & 3 & 8 & 11 \\
Physiotherapy & 1 & 0 & 1 \\
Analgesia + physiotherapy & 0 & 3 & 3 \\
Analgesia + alternative & 0 & 1 & 1 \\
Physiotherapy + alternative & 0 & 1 & 1 \\
\hline
\end{tabular}

Table 8 Number answering correctly

\begin{tabular}{llllll}
\hline & $\begin{array}{l}\text { Ergonomic } \\
\text { training }\end{array}$ & $\begin{array}{l}\text { Operating } \\
\text { height }\end{array}$ & $\begin{array}{l}\text { Monitor } \\
\text { height }\end{array}$ & $\begin{array}{l}\text { Manipulating } \\
\text { angle }\end{array}$ & $\begin{array}{l}\text { Suturing } \\
\text { angle }\end{array}$ \\
\hline Male & Yes & 2 & 1 & 2 & 4 \\
& No & 2 & 1 & 3 & 2 \\
Female & Yes & 0 & 2 & 2 & 2 \\
& No & 3 & 2 & 5 & 4 \\
\hline
\end{tabular}

impact of a number of factors on the likelihood that trainees would report that they had experienced problems with pain or eyestrain following laparoscopic surgery. The model contained seven independent variables (age, sex, height, weight, hand size, years in specialty and ergonomic training).

The full model containing all predictors was not statistically significant, chi-square $(7, N=47)=12.248, p=0.093$, indicating that the model was not able to distinguish between trainees who reported musculoskeletal pain and those who did not. However, a Hosmer-Lemeshow goodness-of-fit test, chi-square $=9.269, p=0.234$, supports the model. The model as a whole explained between 22.9 (Cox \& Snell $R$ square) and $38.3 \%$ (Nagelkerke $R$ square) of the variance in pain status and correctly classified $83 \%$ of cases. Only one of the independent variables made a uniquely statistically significant contribution to the model (height of trainee). The strongest predictor of reporting musculoskeletal pain was gender, recording an odds ratio of 2.19 , indicating that females were over two times more likely to experience pain than their male counterparts. (Table 9)

Again for eyestrain, the full model containing all predictors was not statistically significant, chi-square $(7, N=47)=$ $3.338, p=0.852$, indicating that the model was unable to distinguish between trainees who suffered eyestrain and those who did not. A Hosmer-Lemeshow goodness-of-fit test however (chi-square $=11.218, p=0.129$ ) supports the model. The model as a whole explained only between 6.9 (Cox \& Snell $R$ square) and $9.5 \%$ (Nagelkerke $R$ square)

Table 9 Logistic regression predicting likelihood of reporting musculoskeletal pain

\begin{tabular}{lrrrrrr}
\hline & B & \multicolumn{1}{c}{ S.E. } & Wald & $d f$ & $p$ & Odds ratio \\
\hline Sex & .78 & 2.02 & .15 & 1 & .70 & 2.19 \\
Age & -.08 & .14 & .33 & 1 & .56 & .92 \\
Height & .28 & .13 & 4.63 & 1 & .03 & 1.32 \\
Weight & -.07 & .06 & 1.36 & 1 & .24 & .93 \\
Glove size & -1.47 & 1.15 & 1.65 & 1 & .20 & .23 \\
Years & -.11 & .16 & .47 & 1 & .49 & .90 \\
Ergonomics & -.72 & 1.05 & .47 & 1 & .49 & .49 \\
Constant & -27.50 & 22.54 & 1.489 & 1 & .22 & .000 \\
\hline
\end{tabular}


Table 10 Logistic regression predicting likelihood of reporting eyestrain

\begin{tabular}{lrrrrrl}
\hline & \multicolumn{1}{l}{ B } & \multicolumn{1}{c}{ S.E. } & Wald & $d f$ & $p$ & Odds ratio \\
\hline Sex & .33 & 1.27 & .07 & 1 & .80 & 1.39 \\
Age & -.18 & .17 & 1.19 & 1 & .28 & 0.84 \\
Height & .04 & .06 & .44 & 1 & .51 & 1.04 \\
Weight & -.01 & .04 & .06 & 1 & .80 & 1.00 \\
Glove size & -.11 & .91 & .01 & 1 & .91 & 0.90 \\
Years & .14 & .18 & .62 & 1 & .43 & 1.15 \\
Ergonomics & .35 & .69 & .27 & 1 & .61 & 1.42 \\
Constant & -1.73 & 10.76 & .03 & 1 & .87 & 0.18 \\
\hline
\end{tabular}

of the variance in eyestrain status and correctly classified $66 \%$ of cases. None of the independent variables made a uniquely statistically significant contribution to the model. The strongest predictors for reporting eyestrain were training in ergonomics and gender, recording odds ratios of 1.38 and 1.42 , respectively, indicating that females were over 1.4 times more likely to experience eyestrain than their male counterparts and that trainees who had received training in ergonomics were 1.4 times more likely to suffer eyestrain than those who had not received training (Table 10).

Binary logistic regression analysis was subsequently carried out for each individual region of the body using the same variables.

For the neck, although none of the independent variables made a uniquely statistically significant contribution to the model, female trainees were 7.9 times as likely to experience pain as their male counterparts, while for the back region, none of the independent variables made a uniquely statistically significant contribution to the model, with no variable being any more likely to predict pain than another.

With regard to the shoulder and elbow, none of the independent variables made a uniquely statistically significant contribution to the model.

Table 11 Logistic regression predicting likelihood of reporting wrist pain

\begin{tabular}{lllllll}
\hline & B & S.E. & Wald & $d f$ & $p$ & Odds ratio \\
\hline Sex & .25 & 2.22 & .01 & 1 & .91 & 1.28 \\
Age & -1.31 & .56 & 5.49 & 1 & .02 & .27 \\
Height & -.36 & .15 & 6.16 & 1 & .01 & .70 \\
Weight & .40 & .19 & 4.50 & 1 & .34 & 1.49 \\
Glove size & 1.95 & 1.59 & 1.52 & 1 & .22 & 7.04 \\
Years & 1.32 & .62 & .4 .59 & 1 & .03 & 3.75 \\
Ergonomics & -2.70 & 1.64 & 2.71 & 1 & .10 & .07 \\
Constant & 62.52 & 27.22 & 5.28 & 1 & .02 & $1.42 E+27$ \\
\hline
\end{tabular}

However, for the wrist, the full model containing all predictors was statistically significant, chi-square $=27.83, p=000$, indicating that the model was able to distinguish between trainees who suffered wrist pain and those who did not. Three of the independent variables made a uniquely statistically significant contribution to the model (age, height and years in specialty). The strongest predictor of reporting wrist pain was years' experience recording an odds ratio of 3.75 , indicating that each year spent in specialty led to an almost 4-fold chance of developing wrist pain. (Table 11)

For the thumb, the time that a trainee had spent in specialty was significantly related to the likelihood of suffering pain in that area $(p=0.03)$. Indeed, for each additional year, the likelihood of developing injury rose 1.8 times. For the fingers, the likelihood of developing pain increased 3-fold for each rise in glove size.

For the leg and foot, although none of the independent variables made a uniquely statistically significant contribution to the model, males were 2.7 times more likely to suffer foot pain than their female colleagues.

\section{Conclusions}

This study reports on the prevalence of musculoskeletal distress and eyestrain among trainees in obstetrics and gynaecology.

Sari et al. (2010) reported that less experienced surgeons were more likely to experience injury with laparoscopy than their more experienced colleagues and suggested that this was the result of higher muscle tension and lack of ergonomic knowledge [3]. Indeed, Hemal et al. (2001) noted that surgeons with less than 2 years laparoscopic surgical experience suffered more discomfort than those with greater experience [7].

Thus, instruction in theatre and body ergonomics for laparoscopy early in the medical career should improve the surgical outcomes for both patient and surgeon alike.

Like Stomberg et al. (2010), we found that females were much more likely to suffer musculoskeletal distress than their male colleagues [1]. This difference in prevalence between the sexes has been attributed to the fact that women have a lower muscle mass in the upper extremities when compared with men. As the monitor is often placed on top of a trolley of fixed height, this would disadvantage a shorter surgeon, likely a female whose neck would be extended in viewing the monitor, leading ultimately to neck strain. In addition, as operating tables have traditionally been designed for open surgery, they are not optimal for laparoscopic procedures with the lowest height that most operating tables can be lowered to being only $725 \mathrm{~mm}$, again disadvantaging the usually shorter female surgeon [8]. As our survey shows that female trainees are significantly shorter than their male counterparts, they will 
be automatically exposed to a greater risk of musculoskeletal injury during laparoscopic procedures.

It is somewhat concerning that only a minority of trainees were aware of how to optimise the theatre environment and equipment to minimise the risk of musculoskeletal injury or eyestrain during endoscopic surgery despite all being required to complete a course in Basic Surgical Skills. This would suggest that the current curriculum of the RCOG Basic Surgical Skills course devotes insufficient time and resources to this aspect of surgery and urgently needs to focus more on the ergonomics of both open and endoscopic surgeries if trainees are to minimise their risk of musculoskeletal injury or eyestrain with all the implications that this would have on the specialty.

Moreover, as the retirement age of healthcare workers continues to rise, it is likely that our current cohort of trainees will be forced to work much longer exposing them to an even greater risk of injury unless laparoscopic surgery and the theatre environment are made safer.

There is a potential for such musculoskeletal symptoms to escalate in the future with the increasing application of minimally invasive surgery.

Conflict of interest Declan Quinn and James Moohan declare that they have no conflict of interest.
Consent Informed consent was obtained from all participants included in the study. This article does not contain any studies with human or animal subjects performed by the any of the authors.

\section{References}

1. Stomberg MW, Tronstad S-E, Hedberg K, Bengsston J, Jonsson P, Johansen L, Lindvall B (2010) Work-related musculoskeletal disorders when performing laparoscopic surgery. Surg Laparosc Endosc Percutan Tech 20(1):49-53

2. Szeko GP, Ho P, Ting ACW, Poon JTC, Cheng SWK, Tsang RCC (2009) Work-related musculoskeletal symptoms in surgeons. J Occup Rehabil 19(2):175-184

3. Sari V, Nieboer TE, Vierhout ME, Stegeman DF, Kluivers KB (2010) The operation room as a hostile environment for surgeons: physical complaints during and after laparoscopy. Min Invasiv Ther 19:105-109

4. Soueid A, Oudit D, Thiagarajah S, Laitung G (2010) The pain of surgery: pain experienced by surgeons while operating. Int J Surg 8: $118-120$

5. Park A, Lee G, Seagull FJ, Meenaghan N, Dexter D (2010) Patients benefit while surgeons suffer: an impending epidemic. J Am Coll Surg 210:306-313

6. Cuschieri A (1995) Whither minimal access surgery: tribulations and expectations. Am J Surg 1:9-19

7. Hemal AK, Srinivas M, Charles AR (2001) Ergonomic problems associated with laparoscopy. J Endourol 15:499-503

8. Van Veelen MA, Kazemier G, Koopman J, Goossens RHM, Meijer DW (2002) Assessment of the ergonomically optimal operating surface height for laparoscopic surgery. J Laparoendosc Adv Surg Tech $12: 47-52$ 